\title{
Survival and echocardiographic evaluation of dogs with idiopathic dilated cardiomyopathy treated with carvedilol
}

[Avaliação ecocardiográfica e de sobrevida de cães com cardiomiopatia dilatada idiopática tratados com carvedilol]

\author{
E.C. Soares ${ }^{1}$, G.G. Pereira ${ }^{2}$, L.C. Petrus ${ }^{1}$, M. Leomil Neto ${ }^{3}$, F.L. Yamaki ${ }^{1}$, M.H.M.A. Larsson $^{2}$ \\ ${ }^{1}$ PROVET - Medicina Veterinária Diagnóstica - São Paulo, SP \\ ${ }^{2}$ Faculdade de Medicina Veterinária e Zootecnia - USP - São Paulo, SP \\ Pontifícia Universidade Católica - São Paulo, SP
}

\begin{abstract}
Sixty dogs with idiopathic dilated cardiomyopathy were randomly treated with traditional therapy digitalis, diuretics, angiotensin-converting inhibitors - (group A) or treated with these drugs plus carvedilol (group B). Echocardiographic variables were measured before and after 3, 13, 26, and 52 weeks of treatment or until death. Comparisons between groups and time were performed. No significant differences between groups were found in the most of the echocardiographic variables. The left ventricular end-systolic diameter indexed to body surface area (LVESDi) increased significantly in the group A dogs compared to the group B animals. The survival of groups A and B dogs were not different (P-value=0.1137). In conclusion, the stability of the LVESDi observed in the group treated with carvedilol may represent the beneficial effect over the ventricular remodeling.
\end{abstract}

Keywords: dog, cardiomyopathy, carvedilol, heart failure, echocardiography

\section{RESUMO}

Sessenta cães com cardiomiopatia dilatada idiopática receberam, aleatoriamente, tratamento convencional - digitálicos, diuréticos, inibidores da enzima conversora de angiotensina - (grupo A) ou esses fármacos mais carvedilol (grupo B). As variáveis ecocardiográficas foram avaliadas antes e depois de três, 13, 26 e 52 semanas de tratamento ou até o óbito. Não foram encontradas diferenças significativas entre os grupos de animais quanto à maioria das variáveis ecocardiográficas. O diâmetro sistólico final do ventrículo esquerdo indexado à superfície corpórea (DSVEi) aumentou de forma significativa no grupo A quando comparado ao grupo $B$. Não se observou diferença na sobrevida dos grupos $A$ e $B(P=0,1137)$. Concluiu-se que a estabilização do DSVEi no grupo tratado com carvedilol pode representar o efeito benéfico deste fármaco sobre o remodelamento ventricular.

Palavras-chave: cão, cardiomiopatia, carvedilo, insuficiência cardíaca, ecocardiografia

\section{INTRODUCTION}

Dilated cardiomyopathy (DCM) is the second most common cause of cardiac morbidity and mortality in dogs, mainly among large and giant breeds. The definitive diagnosis is made by echocardiography, in which cardiac chamber dilation and systolic dysfunction are observed. In the last decade, many studies have shown that the diastolic function, as well as systolic, has a very important role in the progression of the disease. Even though the systolic function is the most known determinant of DCM clinical outcome, many human patients with severe contractility impairment show only mild symptoms (Werner et al., 1993).

Some authors (Vanoverscheld et al., 1990; Rihal et al., 1994) demonstrated that the diastolic function is more associated with cardiac failure

Recebido em 19 de novembro de 2009

Aceito em 14 de junho de 2010

E-mail:elainecristina.soares@gmail.com 
functional class. The transmitral flow (TMF) pattern, usually used to assess the diastolic function, is frequently abnormal in DCM and the "restrictive" pattern is correlated to severe disease and is a strong indicator of mortality (Pinamonti et al., 1993). On the other hand, some studies point to systolic function, represented mainly by ejection and shortening fraction, as important predictor of mortality (MacMahon et al., 2004).The classical therapy of DCM is based on diuretics, angiotensin-converting enzyme (ACE) inhibitors, and positive inotropes. Due to the primordial role of sympathetic nervous system in the development and progression of cardiac failure, $\beta$ - blockers have been recently incorporated into the treatment, and carvedilol, a third generation $\beta$-blocker has been heavily investigated. Carvedilol has $\alpha_{1}$ and antioxidant properties, leading to potential benefits of peripheral vasodilation and reduction in apoptosis (Chizzola et al., 2000).

Data have shown the beneficial action of carvedilol on systolic (Chizzola et al., 2000; Opasich et al., 2005) and diastolic function (Palazzuolli et al., 2005), which may be related to an ability of the drug to reverse ventricular remodeling (Opasich et al., 2005). Moreover, favorable effects on survival and symptoms were described by many authors (Packer et al., 1996; Metra et al., 2003).

This study aimed to evaluate the effects of carvedilol on systolic and diastolic functional parameters, ventricular remodeling, and survival in dogs with idiopathic dilated cardiomyopathy.

\section{MATERIAL AND METHODS}

The study groups consisted of 60 dogs presented to the cardiology service at the Veterinary Teaching Hospital due to presence of clinical manifestations related to cardiac disorder or just for screening. Inclusion criteria were shortening fraction less than $25 \%$ and left ventricular Mmode systolic and diastolic dimensions greater than reference values (Boon, 1998). Exclusion criteria were other diseases that could affect the cardiovascular system, mainly renal and endocrine disorders, as well as severe arrhythmias in which antiarrhythmic therapy was primordial. Previous or current treatment with diuretics was allowed.
After idiopathic DCM was diagnosed, each dog was randomly assigned to receive one of the two treatment protocols. The group A dogs protocol $(n=25)$ consisted of diuretics (furosemide: 2$4 \mathrm{mg} / \mathrm{kg}, \quad \mathrm{PO}, \mathrm{q} 8-12 \mathrm{~h}$ and spironolactone: 1$2 \mathrm{mg} / \mathrm{kg}$, PO, q12h), digitalis (digoxin: $0.005 \mathrm{mg} / \mathrm{kg}, \mathrm{PO}, \mathrm{q} 12 \mathrm{~h}$ ) and ACE inhibitors (benazepril: $0.5 \mathrm{mg} / \mathrm{kg}, \mathrm{PO}, \mathrm{q} 24 \mathrm{~h}$ ) and the group $B$ animals protocol $(n=20)$ included all these drugs plus carvedilol $(0.3 \mathrm{mg} / \mathrm{kg}, \mathrm{PO}, \mathrm{q} 12 \mathrm{~h})$. Diuretics were used in cases of congestive heart failure documented by thoracic radiography.

Every dog was submitted to physical examination, blood pressure measurement, complete blood count (CBC) and biochemical profile, ECG, thoracic radiographs, and echocardiography before $\left(\mathrm{T}_{0}\right)$ and after three $\left(\mathrm{T}_{1}\right), 13\left(\mathrm{~T}_{2}\right), 26\left(\mathrm{~T}_{3}\right)$ and $52\left(\mathrm{~T}_{4}\right)$ weeks of therapy or until death. One investigator (ECS) was responsible for clinical and imaging assessment and other (GGP) was responsible for treatment. The dogs were randomized to receive either protocol A or B, in alternating fashion. So, the first dog enrolled received the protocol $\mathrm{A}$, the second received the protocol $\mathrm{B}$, and so on. The first investigator (ECS) was blinded to the protocol administered to each dog.

Cardiopulmonary auscultation was performed on all dogs. The presence of heart murmur was recorded and classified according to the Levine's scale. Abdominal palpation, evaluation of the hydration status by skin elasticity, mucous membranes coloration, and femoral pulse were performed as well.

Each animal was classified according to the International Small Animal Cardiac Health Council heart failure reccomendations, and class IA and IB were considered as "mild" while class II, IIIA, and IIIB were considered as "moderate/severe" heart failure. The systolic blood pressure was recorded by Doppler sphygmomanometry. Three measurements were recorded and the average was considered.

Using an electrocardiography machine Ecafix, model ECG 6, the EKGs were performed with the dog restrained on right lateral recumbency. Unipolar, bipolar, and chest leads were recorded, which provided information about rhythm disturbances, screening for ventricular and supraventricular premature beats, atrial 
fibrillation, and ventricular or supraventricular tachycardia.

Lateral and dorsoventral thoracic radiographs were performed in order to look for cardiomegaly, pulmonary edema, and pleural effusion. The radiographs were done with a CGR machine, model $\mathrm{CH} 300$.

The echocardiographic exams were done with a HITACHI EUB-515-A echocardiography machine, with transducer arrays of $3.5-5.0 \mathrm{MHz}$ and a complete echocardiographic examination, including 2D, M-mode, spectral, and color flow Doppler was done in each dog. The Doppler studies were done from the left apical fourchamber view and included evaluation of transmitral flow (TMF). The studied TMF variables were: $E$-wave peak velocity $\left(\mathrm{E}_{\max }\right)$ and $\mathrm{E}$ wave deceleration time $\left(\mathrm{E}_{\text {dect }}\right)$. From fivechamber view, the left ventricle outflow and mitral inflow profile were recorded at the same time, so the isovolumetric relaxation time (IVRT) could be measured. The recording of the aortic flow allowed the measurement of the stroke volume (SV), as well as the left ventricular ejection time (LVET). The stroke volume was indexed to the body surface area (SVi) and the left ventricular ejection time was corrected to the heart rate, resulting in the left ventricular ejection time index (LVETi) as follows: $\mathrm{LVETi}=\mathrm{LVET}+(0.55 \times \mathrm{HR})$

From short axis views, M-mode measurements included left ventricular end-diastolic diameter (LVEDD), left ventricular end-systolic diameter (LVESD), fractional shortening (FS), left ventricular wall thickness in diastole (LVWTd), left ventricular wall thickness in systole (LVWTs), LVEDD/LVWTd and LVESD/LVWTs ratios, and E-point to septal separation (EPSS). Values of LVEDD and LVESD were indexed to the body surface area (LVEDDi and LVESDi). Data obtained from Mmode measurement and Doppler studies allowed calculation of the velocity of circumferencial shortening (VCF) as: VCF = (LVEDDLVESD)/(LVEDD X LVET). This variable was corrected to the heart rate: $\mathrm{VCFi}=[(\mathrm{VCF} / \mathrm{HR}) \mathrm{X}$ 100]. Data related to echocardiographic measurements are reported as mean \pm standard deviation (SD).
Age, blood pressure, and heart rate homogeneity between the groups at baseline were evaluated by t-student test. The distribution of Dobermans and Boxers in each group was compared by means of chi-square test. The same method was used to compare the incidence of atrial fibrillation, the severity of heart failure and gender of the dogs of groups A and B. When the values were less than 5, the exact Fisher test was used (Armitage and Berry, 2002).

Echocardiographic variables differences were studied considering groups and time. So, group and time effect, as well as the interaction between group and time were evaluated. When the interaction was considered statistically insignificant, group effect (for every time points) and time effect (for both groups) were tested. When the interaction was significant, comparisons between groups were done separately for each time point and the time effect was tested individually for each group. Differences between groups and time points were evaluated by Generalized Estimation Equation with the PROC GENMOD of SAS system. P value less than 0.05 was considered statistically significant.

Survival curves were obtained by the KaplanMeier method. Survival time was counted from the day of diagnosis of DCM to the day of death. The Log-Rank test was applied in order to compare both survival curves (Armitage and Berry, 2002).

Sixty dogs were enrolled in the study. From these, dogs which were still on evaluation at the end of the study [9 (4 - group A; 5 - group B)], those which were lost to follow-up [5 (2- group A; 3 - group B)], and those which died from noncardiac causes ( $1 \mathrm{dog}$ - acute renal failure) were excluded (total $=15$ ).

From the remaining $45 \mathrm{dogs}$ (25 group A, 20 group B), 32 were males and 13 females, averaging ranging from 2 to 13 -year-old, and included several breeds: Great Dane (6), Labrador Retriever (1), Old English Sheepdog (4), German Shepherd (4), Saint Bernard (1), Boxer (6), Cocker Spaniel (8), Doberman Pinscher (7), Neopolitan Mastiff (3), Weimaraner (1), Rottweiller (1). Three were mongrel dogs. Dobermans and Boxers are 
usually more severely affected, and were equally distributed in groups $\mathrm{A}$ and $\mathrm{B}(\mathrm{P}$-value $=0.60)$.

Baseline characteristics including gender, age, blood pressure, heart rate, severity of heart failure, and presence of atrial fibrillation are shown on the Table 1 . No statistical differences between groups A and B were observed with regards to these variables.

Table 1. Values of some echocardiographic variables in dogs with idiopathic dilated not treated (group A) or and treated with carvedilol (group B)

\begin{tabular}{lccc}
\hline \multicolumn{1}{c}{ Variable } & Baseline $\left(\mathrm{T}_{0}\right)$ & P-value \\
& Group A & Group B & \\
\hline Atrial fibrillation & $7 / 25$ & $10 / 20$ & 0.130 \\
Blood pressure $(\mathrm{mmHg})$ & $123 \pm 25$ & $135 \pm 23$ & 0.813 \\
Heart rate $(\mathrm{bpm})$ & $151 \pm 38$ & $155 \pm 49$ & 0.945 \\
Mild heart failure & $4 / 25$ & $3 / 20$ & \\
Moderate/severe heart failure & $21 / 25$ & $17 / 20$ & 0.927 \\
\hline
\end{tabular}

Data obtained during the first evaluation $\left(\mathrm{T}_{0}\right)$, before initiating treatment.

Group A: 7.8 \pm 2.6 -year-old; Group B: 6.9 \pm 2.9 -year-old (P-value= 0.280).

Group A: 17 males and eight females; Group B: 15 males and five females (P-value=0.607).

During the first evaluation $\left(\mathrm{T}_{0}\right)$, pleural effusion was detected in the thoracic radiographs of five dogs of group A and in six dogs of group B; pulmonary edema was seen in seven cases of group A and in five of group B animals, while both (pleural effusion and pulmonary edema) were demonstrated by thoracic radiographies of two dogs of group A and one dog of group B. All dogs had cardiomegaly.

Concerning the results of electrocardiograms performed before initiating the treatment $\left(\mathrm{T}_{0}\right)$, four cases in group A $\operatorname{dogs}$ had isolated ventricular premature complexes (VPC) and the same happened in eight dogs of group B. Atrial premature complexes (APC) were seen in the electrocardiogram of two dogs included in group A. Both (APC and VPC) were detected in two dogs of group A and in three of group B.
Some dogs died during the follow-up period. So, the distribution at each time point was: $\mathrm{T}_{0}=45$ dogs (group $\mathrm{A}=25$; group $\mathrm{B}=20$ ), $\mathrm{T}_{1}=34$ dogs (group $\mathrm{A}=16$; group $\mathrm{B}=18$ ); $\mathrm{T}_{2}=21 \mathrm{dogs}$ (group $A=11 ;$ group $B=10$ ); $T_{3}=9$ dogs (group $A=5$; group $\mathrm{B}=4$ ), and $\mathrm{T}_{4}=7 \operatorname{dogs}$ (group $\mathrm{A}=2$; group $B=5$ ). Due to the few number of observations in the last time point, that could compromise the statistical analysis, $\mathrm{T}_{4}$ was excluded.

Table 2 shows the absolute and percentual number of dogs classified as "mild" and "moderate/severe" heart failure in each time point, considering each group individually. Tables 3a, 3b, and 3c show the obtained echocardiographic values of each studied variable, in each group, represented by mean \pm SD.

Table 2. Number and percentage of severity of heart failures in dogs with idiopathic dilated cardiomyopathy not treat (group A) or treated with carvedilol (group B)

\begin{tabular}{|c|c|c|c|c|c|c|}
\hline \multirow{2}{*}{$\begin{array}{l}\text { Heart } \\
\text { Failure }\end{array}$} & \multicolumn{3}{|l|}{ Group A } & \multicolumn{3}{|l|}{ Group B } \\
\hline & Mild & $\begin{array}{l}\text { Moderate/ } \\
\text { Severe }\end{array}$ & Total & Mild & $\begin{array}{l}\text { Moderate/ } \\
\text { Severe }\end{array}$ & Total \\
\hline $\mathrm{T}_{0}$ & $4(16, \%)$ & $21(84 \%)$ & 25 & $3(15 \%)$ & $17(85 \%)$ & 20 \\
\hline $\mathrm{T}_{1}$ & $8(50, \%)$ & $8(50 \%)$ & 16 & $16(88,9 \%)$ & $2(11.1 \%)$ & 8 \\
\hline $\mathrm{T}_{2}$ & $7(63.6 \%)$ & $4(36.4 \%)$ & 11 & $9(90 \%)$ & $1(10 \%)$ & 10 \\
\hline $\mathrm{T}_{3}$ & $2(40, \%)$ & $3(60 \%)$ & 5 & $3(75 \%)$ & $1(25 \%)$ & 4 \\
\hline $\mathrm{T}_{4}$ & $1(50 \%)$ & $1(50 \%)$ & 2 & $4(80 \%)$ & $1(20 \%)$ & 5 \\
\hline
\end{tabular}

$\mathrm{T}_{0}$ : before treatment; $\mathrm{T}_{1}$ : three weeks after initiating treatment; $\mathrm{T}_{2}: 13$ weeks after initiating treatment; $\mathrm{T}_{3}: 26$ weeks after initiating treatment; $\mathrm{T}_{4}: 56$ weeks after initiating treatment. 
Interaction and group effects were not observed in the statistical analysis of some variables such LVEDDi, LVEDD/LVEWTd, FS, VCFi, Svi, and $E_{\text {dect. }}$ In spite of this, the study of these allowed to notice variations over time regardless the group. So, regarding the variable LVEDDi, a statistically significant difference was not found between groups $\mathrm{A}$ and $\mathrm{B}$ at any one of the time points, but it increased along the time when both groups were considered together. The same occurred with the variables FS, VCFi, Svi, and Edect. On the other hand, the study of the variable LVEDD/LVEWTd demonstrated a decrease over time considering the mean of both groups (Tables 3a, 3b, 3c).

Table 3a. Mean \pm SD of echocardiographic variables of dogs with dilated cardiomyopathy not treated (group A) and treated with carvedilol (group B)

\begin{tabular}{|c|c|c|c|c|c|c|c|c|}
\hline \multirow{2}{*}{$\begin{array}{l}\text { Time } \\
\text { points }\end{array}$} & \multicolumn{2}{|c|}{ LVEDDi $(\mathrm{cm} / \mathrm{m} 2)$} & \multicolumn{2}{|c|}{$\operatorname{LVESDi}(\mathrm{cm} / \mathrm{m} 2)$} & \multicolumn{2}{|c|}{ LVEDD/LVWTd } & \multicolumn{2}{|c|}{ LVESD/LVWTs } \\
\hline & $\mathrm{A}$ & B & $\mathrm{A}$ & B & $\mathrm{A}$ & B & $\mathrm{A}$ & B \\
\hline \multirow[t]{2}{*}{$\mathrm{T}_{0}$} & (25) & (20) & (25) & (20) & (24) & (19) & (24) & (20) \\
\hline & $7.13 \pm 1.52$ & $6.54 \pm 1.62$ & $6.06 \pm 1.41$ & $5.42 \pm 1.26$ & $8.77 \pm 2.53$ & $9.08 \pm 2.83$ & $5.31 \pm 1.48$ & $5.26 \pm 1.20$ \\
\hline \multirow[t]{2}{*}{$\mathrm{T}_{1}$} & (16) & (18) & (16) & (18) & (16) & (18) & (16) & (17) \\
\hline & $7.17 \pm 1.35$ & $6.69 \pm 1.79$ & $5.86 \pm 1.18$ & $5.43 \pm 1.34$ & $8.52 \pm 3.26$ & $8.34 \pm 1.84$ & $5.12 \pm 1.71$ & $4.91 \pm 0.96$ \\
\hline \multirow[t]{2}{*}{$\mathrm{T}_{2}$} & (11) & (10) & (11) & (10) & (11) & (10) & (11) & (10) \\
\hline & $6.99 \pm 1.16$ & $7.07 \pm 1.89$ & $5.66 \pm 0.89$ & $5.66 \pm 1.54$ & $7.77 \pm 3.40$ & $9.48 \pm 2.17$ & $4.64 \pm 1.16$ & $4.89 \pm 1.31$ \\
\hline \multirow[t]{2}{*}{$\mathrm{T}_{3}$} & (5) & (4) & (5) & (4) & (5) & (4) & (5) & (4) \\
\hline & $7.80 \pm 2.04$ & $7.53 \pm 2.58$ & $6.70 \pm 1.50$ & $5.75 \pm 1.56$ & $6.75 \pm 2.97$ & $9.32 \pm 1.15$ & $4.60 \pm 0.89$ & $5.73 \pm 1.40$ \\
\hline
\end{tabular}

$\mathrm{T}_{0}$ : before treatment; $\mathrm{T}_{1}$ : three weeks after initiating treatment; $\mathrm{T}_{2}: 13$ weeks after initiating treatment; $\mathrm{T}_{3}: 26$ weeks after initiating treatment. LVEDDi: left ventricular end-diastolic diameter indexed to body surface area; LVESDi: left ventricular end-systolic diameter indexed to body surface area; LVEDD/LVWTd: ratio between left ventricular enddiastolic diameter and left ventricular wall thickness in diastole; LVESD/LVWTs: ratio between left ventricular endsystolic diameter and left ventricular wall thickness in systole.

Table 3b. Mean \pm SD of echocardiographic variables of dogs with dilated cardiomyopathy not treated (group A) and treated with carvedilol (group B)

\begin{tabular}{ccccccccc}
\hline Time & \multicolumn{2}{c}{ FS $(\%)$} & \multicolumn{2}{c}{ EPSS $(\mathrm{cm})$} & \multicolumn{2}{c}{ LVETi (ms) } & \multicolumn{2}{c}{ VCFi (circ/s) } \\
\cline { 2 - 8 } points & $\mathrm{A}$ & $\mathrm{B}$ & $\mathrm{A}$ & $\mathrm{B}$ & $\mathrm{A}$ & $\mathrm{B}$ & $\mathrm{A}$ & $(20)$ \\
\hline $\mathrm{T}_{0}$ & $(25)$ & $(20)$ & $(25)$ & $(20)$ & $(25)$ & $(20)$ & $(25)$ & $(20)$ \\
& $15.58 \pm 0.27$ & $16.34 \pm 4.80$ & $2.13 \pm 1.04$ & $1.96 \pm 0.60$ & $222.04 \pm 36.74$ & $226.70 \pm 19.28$ & $0.79 \pm 0.42$ & $0.71 \pm 0.24$ \\
$\mathrm{~T}_{1}$ & $(16)$ & $(18)$ & $(16)$ & $(18)$ & $(16)$ & $(18)$ & $(16)$ & $(18)$ \\
& $17.92 \pm 4.76$ & $18.54 \pm 4.60$ & $1.87 \pm 0.70$ & $1.88 \pm 0.42$ & $227.63 \pm 19.39$ & $233 \pm 24.21$ & $0.92 \pm 0.46$ & $0.91 \pm 0.29$ \\
$\mathrm{~T}_{2}$ & $(11)$ & $(10)$ & $(11)$ & $(10)$ & $(11)$ & $(10)$ & $(11)$ & $(10)$ \\
& $18.38 \pm 3.67$ & $20.16 \pm 7.52$ & $1.73 \pm 0.45$ & $1.84 \pm 0.75$ & $225.45 \pm 21.28$ & $241.60 \pm 18.89$ & $0.99 \pm 0.27$ & $1.03 \pm 0,45$ \\
$\mathrm{~T}_{3}$ & $(5)$ & $(4)$ & $(5)$ & $(4)$ & $(5)$ & $(4)$ & $(5)$ & $(4)$ \\
& $18.08 \pm 3.72$ & $19.95 \pm 7.90$ & $1,63 \pm 0.68$ & $2.55 \pm 1.45$ & $227.80 \pm 7.56$ & $218.75 \pm 22.60$ & $1.01 \pm 0.28$ & $1.27 \pm 0.46$ \\
\hline
\end{tabular}

$\mathrm{T}_{0}$ : before treatment; $\mathrm{T}_{1}$ : three weeks after initiating treatment; $\mathrm{T}_{2}: 13$ weeks after initiating treatment; $\mathrm{T}_{3}: 26$ weeks after initiating treatment. FS: fractional shortening; EPSS: E-point to septal separation; LVETi: left ventricular ejection time corrected to heart rate; VCFi: velocity of circumferential shortening corrected to heart rate.

Table 3c. Mean \pm SD of echocardiographic variables of dogs with dilated cardiomyopathy not treated (group A) and treated with carvedilol (group B).

\begin{tabular}{|c|c|c|c|c|c|c|c|c|}
\hline \multirow{2}{*}{$\begin{array}{l}\text { Time } \\
\text { points }\end{array}$} & \multicolumn{2}{|c|}{$\mathrm{SVi}(\mathrm{mL} / \mathrm{m} 2)$} & \multicolumn{2}{|c|}{$\mathrm{E}_{\text {max }}(\mathrm{m} / \mathrm{s})$} & \multicolumn{2}{|c|}{$\mathrm{E}_{\text {dect }}(\mathrm{ms})$} & \multicolumn{2}{|c|}{ IVRT (s) } \\
\hline & $\mathrm{A}$ & $\mathrm{B}$ & $\mathrm{A}$ & $\mathrm{B}$ & $\mathrm{A}$ & B & A & B \\
\hline \multirow[t]{2}{*}{$\mathrm{T}_{0}$} & (25) & (20) & (25) & (20) & (25) & (20) & (25) & (20) \\
\hline & $18.31 \pm 9.03$ & $18.60 \pm 9.48$ & $0.89 \pm 0.27$ & $0.97 \pm 0.34$ & $88.90 \pm 26.49$ & $88.75 \pm 19.75$ & $0.07 \pm 0.03$ & $0.07 \pm 0.03$ \\
\hline \multirow[t]{2}{*}{$\mathrm{T}_{1}$} & (16) & (18) & (16) & (18) & (15) & $(17)$ & (16) & (18) \\
\hline & $22 \pm 8.65$ & $16.90 \pm 9.53$ & $1.01 \pm 0.29$ & $0.92 \pm 0.29$ & $95.40 \pm 28.40$ & $96.35 \pm 23.58$ & $0.07 \pm 0.04$ & $0.07 \pm 0.03$ \\
\hline \multirow[t]{2}{*}{$\mathrm{T}_{2}$} & (11) & (10) & (11) & (10) & (11) & (10) & (11) & (10) \\
\hline & $23.39 \pm 8.99$ & $19.90 \pm 9.07$ & $0.97 \pm 0.26$ & $0.97 \pm 0.34$ & $97.68 \pm 28.92$ & $104.8 \pm 34.19$ & $0.07 \pm 0.03$ & $0.06 \pm 0.03$ \\
\hline \multirow[t]{2}{*}{$\mathrm{T}_{3}$} & (5) & (4) & (5) & (4) & (5) & (4) & (5) & (4) \\
\hline & $24.60 \pm 9.41$ & $28.15 \pm 12.27$ & $0.86 \pm 0.22$ & $0.85 \pm 0.31$ & $108 \pm 28.04$ & $92.25 \pm 20.84$ & $0.07 \pm 0.04$ & $0.11 \pm 0.05$ \\
\hline
\end{tabular}

$\mathrm{T}_{0:}$ before treatment; $\mathrm{T}_{1}$ : three weeks after initiating treatment; $\mathrm{T}_{2}: 13$ weeks after initiating treatment; $\mathrm{T}_{3}: 26$ weeks after initiating treatment. Svi: stroke volume indexed to body surface area; $\mathrm{E}_{\max }$ : $\mathrm{E}$ wave maximal velocity; $\mathrm{E}_{\text {dect }}$ : $\mathrm{E}$ wave deceleration time; IVRT: isovolumetric relaxation time. 
The analysis of the variables LVESD/LVWTs, LVETi, $\mathrm{E}_{\max }$ and IVRT failed to demonstrate interaction, group or time effect, i.e, no differences were observed between groups or along the time. Significance levels related to the study of these variables - along the time and independently of group - are described in the Table 4. Others, as LVESDi and EPSS showed interaction and group effects. LVESDi increased significantly over the time in group $\mathrm{A}\left(\mathrm{T}_{0} \mathrm{X}\right.$ $\mathrm{T}_{3}=0.0459 ; \mathrm{T}_{1} \times \mathrm{T}_{3}=0.0066 ; \mathrm{T}_{2} \times \mathrm{T}_{3}=0.0007$ ) what did not occur in the group $\mathrm{B}$.

Table 4. P-values of the comparisons between time points of echocardiographic variables considering the mean of both groups (A and B)

\begin{tabular}{ccccccccccc}
\hline $\begin{array}{c}\text { Comparison } \\
\text { between time } \\
\text { points }\end{array}$ & $\begin{array}{c}\text { LVEDDi } \\
\left(\mathrm{cm} / \mathrm{m}^{2}\right)\end{array}$ & $\begin{array}{c}\text { LVEDD/ } \\
\text { LVWTd }\end{array}$ & $\begin{array}{c}\text { LVESD/ } \\
\text { LVWTs }\end{array}$ & $\begin{array}{c}\text { FS } \\
(\%)\end{array}$ & $\begin{array}{c}\text { LVETi } \\
(\mathrm{ms})\end{array}$ & $\begin{array}{c}\text { VCFi } \\
(\mathrm{circ} / \mathrm{s})\end{array}$ & $\begin{array}{c}\mathrm{SVi} \\
\left(\mathrm{mL} / \mathrm{m}^{2}\right)\end{array}$ & $\begin{array}{c}\mathrm{E}_{\max } \\
(\mathrm{m} / \mathrm{s})\end{array}$ & $\begin{array}{c}\mathrm{E}_{\text {dect }} \\
(\mathrm{ms})\end{array}$ & $\begin{array}{c}\text { IVRT } \\
(\mathrm{s})\end{array}$ \\
\hline $\mathrm{T}_{0} \mathrm{X} \mathrm{T}_{1}$ & 0.9455 & 0.2262 & 0.3997 & $\mathbf{0 . 0 0 1 2}$ & 0.2560 & $\mathbf{0 . 0 1 8 1}$ & 0.5673 & 0.6543 & $\mathbf{0 . 0 1 4 5}$ & 0.4836 \\
$\mathrm{~T}_{0} \mathrm{X} \mathrm{T}_{2}$ & 0.2999 & 0.6881 & 0.0890 & $\mathbf{0 . 0 0 1 0}$ & 0.1349 & $\mathbf{0 . 0 0 1 1}$ & 0.3028 & 0.3927 & $\mathbf{0 . 0 4 5 8}$ & 0.2373 \\
$\mathrm{~T}_{0} \mathrm{X} \mathrm{T}_{3}$ & $\mathbf{0 . 0 0 0 8}$ & $\mathbf{0 . 0 2 5 7}$ & 0.4253 & $\mathbf{0 . 0 0 7 0}$ & 0.9935 & $\mathbf{0 . 0 0 0 7}$ & $\mathbf{0 . 0 2 3 5}$ & 0.1470 & 0.1380 & 0.5531 \\
$\mathrm{~T}_{1} \mathrm{X} \mathrm{T}_{2}$ & 0.1374 & 0.4721 & 0.3306 & 0.2404 & 0.6609 & 0.1525 & 0.4716 & 0.6329 & 0.2545 & 0.4505 \\
$\mathrm{~T}_{1} \mathrm{X} \mathrm{T}_{3}$ & $\mathbf{0 . 0 0 0 6}$ & 0.2761 & 0.8688 & 0.3300 & 0.3611 & 0.0573 & 0.0734 & 0.0681 & 0.4080 & 0.3627 \\
$\mathrm{~T}_{2} \mathrm{X} \mathrm{T}_{3}$ & $\mathbf{0 . 0 0 1 5}$ & 0.0744 & 0.6742 & 0.8439 & 0.2356 & 0.3525 & 0.2110 & 0.0568 & 0.8780 & 0.2407 \\
\hline
\end{tabular}

$\mathrm{T}_{0}$ : before treatment; $\mathrm{T}_{1}$ : three weeks after initiating treatment; $\mathrm{T}_{2}: 13$ weeks after initiating treatment; $\mathrm{T}_{3}: 26$ weeks after initiating treatment. LVEDDi: left ventricular end-diastolic diameter indexed to body surface area; LVEDD/LVWTd: ratio between left ventricular end-diastolic diameter and left ventricular wall thickness in diastole; LVESD/LVWTs: ratio between left ventricular end-systolic diameter and left ventricular wall thickness in systole; FS: fractional shortening; LVETI: left ventricular ejection time corrected to heart rate; VCFi: velocity of circumferencial shortening corrected to heart rate; $\mathrm{SVi}$ : stroke volume indexed to body surface area; $\mathrm{E}_{\max }$ : $\mathrm{E}$ wave maximal velocity; $\mathrm{E}_{\text {dect }}: \mathrm{E}$ wave deceleration time; IVRT: isovolumetric relaxation time. $\mathrm{P}<0.05$

There was a decrease in EPSS mean value of the group $\mathrm{A}\left(\mathrm{T}_{0} \mathrm{X} \mathrm{T}_{2}=0.0148 ; \mathrm{T}_{0} \mathrm{X} \mathrm{T}_{3}=0.0045\right)$, but in the group $\mathrm{B}$ the mean value of this variable increased $\left(\mathrm{T}_{2} \mathrm{X} \mathrm{T}_{3}=0.0372\right)$.

Considering both groups, the median survival time was 136 days (95\%CI=99-173). The dogs of group A had a median survival time of 113 days (95\% CI=68-159), while those included in the group B survived for a median time of 164 days (95\% CI=107-221). The comparison of survival curves (Figure 1) failed to demonstrate significant difference between group A and B (Pvalue $=0.1137$ ) .

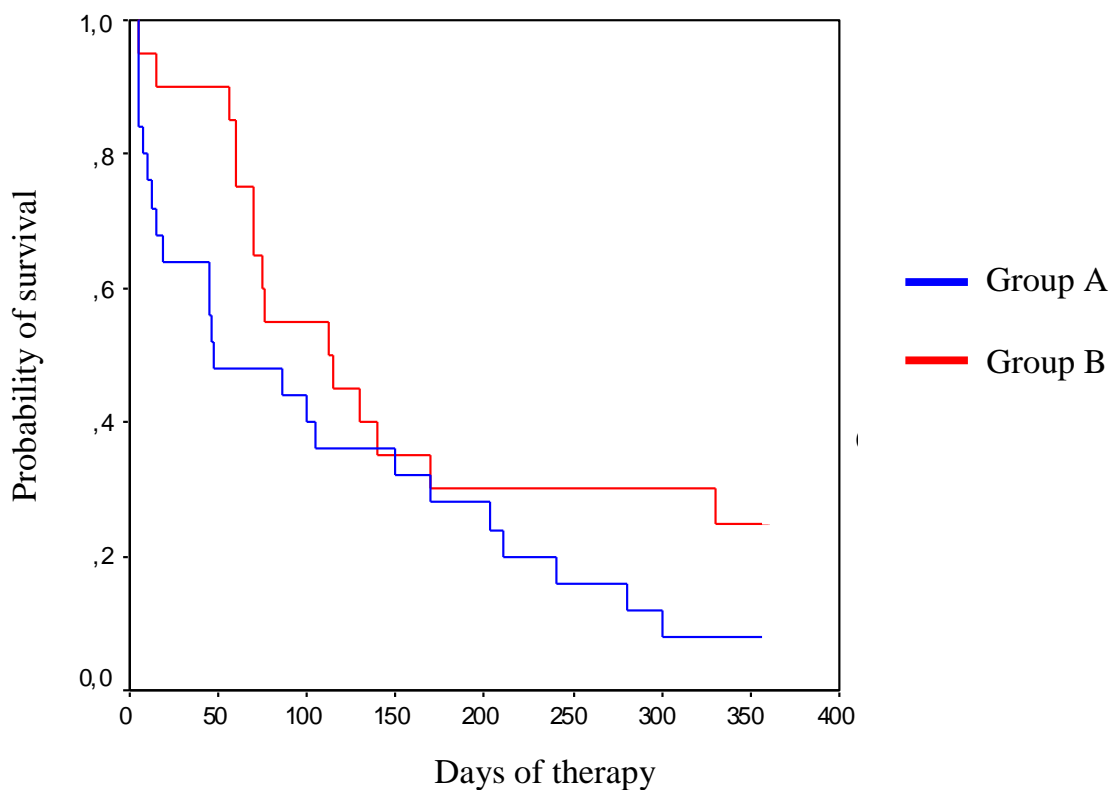

Figure 1. Survival curves (Kaplan-Meier) of dogs with idiopathic dilated cardiomyopathy not treated (group A) and treated (group B) with carvedilol. 


\section{DISCUSSION}

The fact that the group of dogs not treated with carvedilol showed increase in the LVESDi over the time, while the same did not happen in the group treated with carvedilol can be interpreted as an attenuattion effect of this drug on ventricular remodeling.

Many authors have described the benefits of carvedilol on ventricular function and remodeling in human patients with ischemic and non-ischemic cardiomyopathy. Palazzuolli et al. (2005), studying the action of carvedilol on human patients with DCM, observed an increase of the ejection fraction as well as a reduction in the left ventricular end systolic volume after a year of therapy. Kotlyar et al. (2004) also related LVEDD and LVESD reduction, but only after two years of treatment. Chizzola et al. (2000) studied human patients with DCM during a sixmonth follow-up period and noticed an increase in FS and EF in the group submitted to carvedilol therapy. Nevertheless, the authors did not observe changes in ventricular diameter. On the other hand, a rise of the systolic and diastolic ventricular volume was related by Quaife et al. (1996) in a study involving human patients with idiopathic DCM and ischemic cardiomyopathy (1996).

Remme et al. (2004) and Rahko (2005) described the reversal of ventricular remodeling in people treated with carvedilol and this effect was always accompanied by elevation of the FS and EF. Recently, Oyama et al. (2007) failed to demonstrate any improvement on ventricular volume or systolic function in dogs with DCM treated with carvedilol over a four-month treatment period.

Concerning about the action of the drug on the diastolic function, Palazzuolli et al. (2005) observed an improvement of ventricular relaxation pattern after four months of therapy in people with advanced heart failure. Bergström et al. (2004) also noticed E/A ratio normalization. Therefore, significant changes of $\mathrm{E}_{\text {dect }}$ and IVRT were not verified. Cappomolla et al. (2000) related that the E/A ratio of human patients put on carvedilol changed to a "less restrictive" pattern, i.e, the A wave peak velocity increased while the $\mathrm{E}$ wave peak velocity decreased, and the $\mathrm{E}_{\mathrm{dect}}$ became longer.
The lack of statistical significant differences in the other echocardiographic variables between groups A and B can be explained by the small number of dogs, mainly at the last time point studied. Many dogs were enrolled in "moderate/severe" heart failure and so death occurred early and frequently. Due to such a small study population the standard deviation had a broad range, what could lead to statistically insignificant results. Besides that, most studies in human medicine use a gradually increasing dose of $\beta$-blockers, which was not done in our research. The conservative dose of $0.3 \mathrm{mg} / \mathrm{kg}$, PO, q12h, was chosen according to Uechi et al. (2002). These authors studied the hemodynamic and renal effects of carvedilol in healthy and cardiac dogs and observed that a dose of $0.4 \mathrm{mg} / \mathrm{kg}$ was enough to cause renal function deterioration. Yet, according to them, $\beta$-blocking action could be observed with a dose so low as $0.2 \mathrm{mg} / \mathrm{kg}$. So, it was decided to use an intermediate dose. Moreover, many dogs included in the study suffered from severe heart failure and there was not certainty about the safety of prescribing a higher dose in these patients. Nevertheless, the low dose may be responsible for the insignificant results.

Some echocardiographic variables changed over the time, regardless of group. So, the mean value of the variable LVEDD/LVWTd decreased after 52 weeks of therapy. As LVEDD rose up along the time, it may be concluded that this change might have happened due to a larger increase of LVWTd.

The elevation of variables related to systolic function, as FS and VCFi, might have occurred because of positive inotropic action of digoxin, as well as afterload reduction caused by ACE inhibitor and diuretics. Moreover, it was observed a larger increase of LVEDD compared to the increase in LVESD and this could be partially responsible for the elevation of FS.

Concerning diastolic function, the $\mathrm{E}_{\text {dect }}$ was the only variable that suffered a significant change over the time, increasing in both groups at 26 weeks of therapy. That could point to ventricular relaxation improvement but as it occurred independently of group, it could not ensure that carvedilol accounted for this change. ACE inhibitors, as well as carvedilol, have vasodilatatory properties that could have reduced 
intraventricular pressure in association with the $\mathrm{E}_{\text {dect }}$ increase.

About $38 \%$ of dogs were in atrial fibrillation, damaging substantially the analysis of the transmitral inflow. However, this arrhythmia is frequently observed in the natural occurring DCM, so the exclusion of dogs with atrial fibrillation of the study could significantly reduce the sample size. Moreover, the importance of a clinical research like this consists in the evaluation of the response to the drug in the natural disease.

This research demonstrated that the dogs treated with carvedilol had a survival time $45 \%$ longer than those which did not receive the drug. Nevertheless, the statistical analysis failed to show favorable effects of carvedilol over survival, the careful observation of Kaplan-Meier curves allows to conclude that dogs not treated with carvedilol died earlier. Undoubtedly, this fact has a very important clinical relevance.

The most of the dogs included in this study had advanced heart failure; therefore, many of them died early during the follow-up period. As the benefits of $\beta$-blockers are generally observed after three months of therapy, many dogs died before a positive response could be noticed.

Probably, a larger study population, the use of a higher dose as well as the up-titration could lead to more significant results, with important differences between carvedilol treated and not treated groups.

\section{ACKNOWLEDGMENTS}

The authors thank FAPESP (Fundação de Amparo a Pesquisa do Estado de São Paulo) for all the financial support and to NOVARTIS for providing Fortekor® (Benazepril Chloridrate).

\section{REFERENCES}

ARMITAGE, P., BERRY, G. Statistical methods in medical research. 3.ed. Oxford: Blackwell Science, 1994.

BOON, J.A. Manual of veterinary echocardiographs. Baltimore: Williams \& Wilkins, 1998. 478p.
CAPOMOLLA, S.; FEBO, O.; GNEMMI, M. et al. $\beta$-blockade therapy in chronic heart failure: diastolic function and mitral regurgitation improvement by carvedilol. Am. Heart J., v.139, p.596-608, 2000.

CHIZZOLA, P.R.; FREITAS, H.F.G.; CALDAS, M.A. et al. Efeitos do carvedilol na insuficiência cardíaca por cardiomiopatia dilatada. Resultado de estudo duplo-cego, randômico, com grupo placebo-controle (estudo CARIBE). Arq. Bras. Cardiol., v.74, p.233-237, 2000.

KOTLYAR, E.; HAYWARD, C.S.; KEOGH, A.M. et al. The impact of baseline left ventricular size and mitral regusgitation on reverse left ventricular remodeling in response to carvedilol: size doesn't matter. Heart, v.90, p.800-801, 2004.

MCMAHON, C.J.; NAGUEH, S.F.; EAPEN, R.S. et al. Echocardiographic predictors of adverse clinical events in children with dilated cardiomyopathy: a prospective clinical study. Heart, v.90, p.909-915, 2004.

METRA, M.; NODARI, S.; PARRINELLO, G. et al. Marked improvement in left ventricular ejection fraction during long-term $\beta$-blockade in patients with chronic heart failure: clinical correlates and prognostic significance. Am. Heart J., v.145, p.292-299, 2003.

OPASICH, C.; De FEO, S.; CIOFFI, G. et al. Does atrial fibrillation in elderly patiens with chronic heart failure limit the efficacy of carvedilol? Suggestions from an observational study. Ital. Heart J., v.6, p.323-327, 2005.

OYAMA M.A; SISSON D.D; PROSEK R. Carvedilol in dogs with dilated cardiomyopathy. J. Vet. Intern. Med., v.21, p.1272-1279, 2007.

PACKER, M.; BRISTOW, M.R.; COHN, J.N. et al. The effect of carvedilol on morbidity and mortality in patients with chronic heart failure. N. Engl. J. Med., v.334, p.1349-1355, 1996.

PALAZZUOLI, A.; QUATRINI, I; VECCHIATO, L. et al. Left ventricular diastolic function improvement by carvedilol therapy in advanced heart failure. J. Cardiovasc Pharmacol., v.45, p.563-568, 2005. 
PINAMONTI, B.; DI LENARDA, A.; SINAGRA, G. et al. Restrictive left ventricular filling pattern in dilated cardiomyopathy assessed by doppler echocardiography: clinical, echocardiographic and hemodynamic correlations and prognostic implications. J. Am. Coll. Cardiol., v.22, p.808-815, 1993.

QUAIFE, R.A.; GILBERT, E.M.; CHRISTIAN, P.E. et al. Effects of carvedilol on systolic and diastolic left ventricular performance in idiopathic dilated cardiomyopathy or ischemic cardiomyopathy. Am. J. Cardiol., v.78, p.779784, 1996.

RAHKO, P.S. An echocardiographic analysis of the long-term effects of carvedilol on left ventricular filling patterns in dilated cardiomyopathy. Echocardiography, v.22, p.547-554, 2005.

REMME, W.J.; RIEGGER, G.; HILDEBRANDT, P. et al. The benefits of early combination treatment of carvedilol and an ACE-inhibitor in mild heart failure and left ventricular systolic dysfunction. The carvedilol and ACE-inhibiton remodelling mild heart failure evaluation trial (CARMEN). Cardiovasc. Drugs Ther., v.18, p.57-66, 2004.
RIHAL, C.S.; NISHIMURA, R.A.; HATLE, L.K. et al. Congestive heart failure/IV hipertrophy: systolic and diastolic dysfunction in patients with clinical diagnosis of dilated cardiomyopathy: relation to symptoms and prognosis. Circulation, v.90, p.2772-2779, 1994.

UECHI, M.; SASAKI, T.; UENO, K. et al. Cardiovascular and renal effects of carvedilol in dogs with heart failure. J. Vet. Med. Sci., v.64, p.469-475, 2002.

VANOVERSCHED， J.J.; RAPHAEL， D.A.; ROBERT, A.R. et al. Left ventricular filling in dilated cardiomyopathy: relation to functional class and hemodynamics. J. Am. Coll. Cardiol., v.15, p.1288-1295, 1990.

WERNER, G.S.; SCHAEFER, C.; DIRKS, R. et al. Doppler echocardiographic assessment of left ventricular filling in idiopathic dilated cardiomyopathy during a one-year follow-up: relation to the clinical course of disease. Am. Heart J., v.126, p.1408-1416, 1993. 